\title{
Clinical Criteria for Tracheostomy Decannulation in Subjects with Acquired Brain Injury
}

\author{
Claudia Enrichi MSc, Irene Battel MSc, Cristiano Zanetti MSc, Isabella Koch MSc, \\ Laura Ventura PhD, Katie Palmer PhD, Francesca Meneghello MD, Francesco Piccione MD, \\ Simonetta Rossi MSc, Marta Lazzeri MSc, Maurizio Sommariva PT, and Andrea Turolla PhD
}

BACKGROUND: Patients with acquired brain injury (ABI) often require long periods of having a tracheostomy tube for airway protection and prolonged mechanical ventilation. It has been recognized that fast and safe decannulation improves outcomes and facilitates the recovery process. Nevertheless, few studies have provided evidence for decannulation criteria, despite the high prevalence of ABI subjects with tracheostomies. The aim of our study was to assess which clinical parameters are the best predictors for decannulation in subjects with ABI. METHODS: In this cross-sectional study, we recruited 74 consecutive ABI subjects (mean age $51.52 \pm 16.76$ ) with tracheostomy tubes. First, the subjects underwent the original decannulation assessment for cannula removal. Second, they underwent our experimental decannulation protocol. The experimental protocol included: voluntary cough (cough peak flow $\geq 160 \mathrm{~L} / \mathrm{min}$ ), reflex cough, tracheostomy tube capping ( $\geq 72 \mathrm{~h}$ ), swallowing instrumental assessment (penetration aspiration scale $\leq 5$ ), blue dye test, number of trachea suctions, endoscopic assessment of airway patency (lumen diameter $\geq 50 \%$ ), saturation $\left(\mathrm{S}_{\mathrm{pO2}}>95 \%\right)$, and level of consciousness evaluation (Glasgow coma scale $\geq 8$ ). The reference standard was clinical removal of the tracheostomy tube within $48 \mathrm{~h}$. RESULTS: Parameters showing the highest values of sensitivity and specificity, respectively, were tracheostomy tube capping $(80 \%, 100 \%)$, endoscopy assessment of airway patency $(100 \%, 30 \%)$, swallowing instrumental assessment $(85 \%, 96 \%)$, and the blue dye test $(65 \%, 85 \%)$. All these were combined in a clinical cluster parameter, which had higher sensitivity $(\mathbf{1 0 0 \%})$ and specificity $(\mathbf{8 2 \%})$. CONCLUSION: These results suggest that the best clinical prediction rule for decannulation in acquired brain injury subjects is a combination of the following assessments: (1) tracheostomy tube capping, (2) endoscopic assessment of patency of airways, (3) swallowing instrumental assessment, and (4) blue dye test. Key words: acquired brain injury; tracheostomy tube; decannulation protocol; weaning protocol; dysphagia; voluntary cough; reflex cough; blue dye test; airways patency; tracheostomy tube capping. [Respir Care 2017;62(10):1255-1263. (C) 2017 Daedalus Enterprises]

\section{Introduction}

Tracheostomy is one of the most frequent procedures in intensive care unit patients: about $10 \%$ of patients requir-

Claudia Enrichi, Irene Battel, Cristiano Zanetti, Isabella Koch, Katie Palmer, Francesca Meneghello, Francesco Piccione, Simonetta Rossi, and Andrea Turolla are affiliated with Fondazione Ospedale di Neuroriabilitazione, Istituto di Ricovero e Cura a Carattere Scientifico, San Camillo, Venice, Italy.

Ms Lazzeri and Mr Sommariva are affiliated with Ospedale Niguarda Ca' Granda, Milan, Italy. ing more than $3 \mathrm{~d}$ of mechanical ventilation are expected to undergo tracheostomy. ${ }^{1,2}$ Although the removal of tra-

\footnotetext{
Laura Ventura is affiliated with Dipartimento di Scienze StatisticheUniversita' di Padova, Padova, Italy.

The authors have disclosed no conflicts of interest.

Correspondence: Claudia Enrichi, Fondazione Ospedale di Neuroriabilitazione IRCCS San Camillo, Via Alberoni n ${ }^{\circ} 70$, postcode 30126, Venice, Italy. E-mail: claudia.enrichi@ospedalesancamillo.net.
}

DOI: $10.4187 /$ respcare. 05470 
cheostomy tubes (decannulation) is not risk-free, there is evidence of benefits from decannulation. ${ }^{2,3}$ Keeping a tracheostomy tube in place may cause inflammation and stenosis or excessive cough, and it may impair swallowing by impeding tracheal elevation against the epiglottis, which is an automatic mechanism to prevent aspiration of food or secretions. ${ }^{3,4}$

The frequency of tracheostomy in patients with traumatic brain injury contrasts with the lack of objective criteria for weaning. In the literature, there are different decannulation protocols designed mostly for acute intensive care where tracheostomy tubes are expected to be removed after a short time period. ${ }^{1,5}$ Conversely, there is a lack of information on this topic concerning postacute patients with acquired brain injury (ABI). ${ }^{6}$ This population is characterized not only by severe neurological impairment but also by cognitive disorders, which alter motor control and the ability to execute simple voluntary tasks. In addition, these patients often have tracheostomy tubes for a long period of time, which increases the risk of adverse effects such as formation of granulation tissue, tracheal stenosis, or tracheomalacia. ${ }^{4,7,8}$ It is widely accepted that there are clear benefits to tracheostomy tube removal. ${ }^{3}$ Hence, there is a growing need to identify a protocol not only for assessing whether a patient is ready for decannulation but also specifically designed for ABI patients. A recent systematic review of tracheostomy decannulation by Santus et $\mathrm{al}^{6}$ selected 2 quantitative and 8 semi-quantitative criteria for safe tube removal. Although they emphasized the importance of defining specific criteria, the majority of them were subjective, depending upon the experience of the healthcare professionals.

The aims of the our study were to test the performance of quantitative parameters for safe decannulation in postacute $\mathrm{ABI}$ subjects and to explore whether the clustering of more than one test might result in a better clinical prediction rule for safe decannulation of $\mathrm{ABI}$ subjects.

\section{Methods}

This cross-sectional study was conducted in accordance with the amended Declaration of Helsinki and received approval from the ethical committee for clinical experimentation of the province of Venice and Istituto di Ricovero e Cura a Carattere Scientifico. Informed consent was obtained from all participants directly or from legal decision makers or proxies, according to their ability to provide informed consent. All participants were assessed first for decannulation following the original decannulation protocol, and the decision for cannula removal was based on this protocol. Second, they underwent the experimental decannulation protocol. Those who assessed subjects according to the decannulation protocols were blinded to the experimental outcome to avoid bias during the pro-

\section{QUICK LOOK}

\section{Current knowledge}

Decannulation protocols are designed mostly for intensive care, thus tracheostomy tubes are expected to be removed after a short time. There is a lack of information on this topic concerning postacute subjects with acquired brain injury (ABI). $\mathrm{ABI}$ is characterized by severe neurological impairments and cognitive disorders, which alter motor control and ability to execute simple voluntary tasks. Thus, decannulation protocols for intensive care might be not adequate for ABI decannulation.

\section{What this paper contributes to our knowledge}

This study investigated the most significant and objective parameters for tube removal in postacute ABI subjects. From our findings, four parameters showed adequate sensitivity and specificity to be clustered as a unique diagnostic test for weaning from tracheostomy tube among postacute ABI subjects.

cess of decannulation. Finally, participants underwent follow-up assessment after $48 \mathrm{~h}$ to verify whether the tracheostomy tube was removed, which was considered the reference standard.

\section{Subjects}

Participants were consecutively recruited immediately after admission to the Neurorehabilitation Department of Fondazione Ospedale San Camillo in Venice, Italy, from July 2, 2015, to July 31,2016 . The inclusion criteria were confirmed diagnosis of $\mathrm{ABI}$, presence of tracheostomy cannula, absence of citrus allergy, age $>18$ years, and clinical stability defined as the absence of fever, sepsis, or active infection and hemodynamic stability. ${ }^{9}$

\section{Decannulation Protocol}

This protocol was completed by the senior neurologist and a speech and language therapist, who were responsible for making decisions concerning decannulation. The decision for decannulation was based on the following parameters, which have been described in the literature:

1. Toleration of tracheostomy tube capping for $72 \mathrm{~h} .{ }^{10}$

2. Absence of severe dysphagia, defined as the ability to manage secretions, which was assessed by a senior speech and language therapist. ${ }^{11,12}$ 


\section{Clinical Criteria for Tracheostomy Decannulation}

Table 1. Summary of the Parameters of Experimental Decannulation Protocol, Description of the Modality of Assessment, and Relative Criteria for Positivity

\begin{tabular}{|c|c|c|}
\hline Parameters & Modality of Assessment & Criteria for Positivity \\
\hline Voluntary cough & Spirometer (Pony FX, Cosmed, Rome, Italy) & Cough peak flow $\geq 160 \mathrm{~L} / \mathrm{min}$ \\
\hline Reflex cough & $\begin{array}{l}\text { Inhalation of } 0.1 \mathrm{~mol} / \mathrm{L} \text { citric acid using MO-03 } \\
\text { nebulizer (Norditalia Eletromedicali, San } \\
\text { Martino della Battaglia, Italy) }\end{array}$ & Presence of 2 consecutive coughs \\
\hline Tracheostomy tube capping & $\begin{array}{l}\text { Ability to breathe through the nose and mouth } \\
\text { with tracheostomy tube capped }\end{array}$ & Tracheostomy tube capping $\geq 72 \mathrm{~h}$ \\
\hline Swallowing instrumental assessment & $\begin{array}{l}\text { Fibroendoscopic evaluation during liquid and food } \\
\text { administration; severity score assessed by } \\
\text { penetration assessment scale }\end{array}$ & $\begin{array}{l}\text { Penetration assessment scale } \leq 5 \text {, no } \\
\text { aspiration events }\end{array}$ \\
\hline Blue dye test & Trachea suctioned every $4 \mathrm{~h}$ during a 12 -h interval & Absence of blue traces \\
\hline Number of tracheal suctions & Nurses wrote down the number of suctions & $\leq 2$ suctions every $8 \mathrm{~h}$ \\
\hline Endoscopic assessment of airway patency & Endoscopy evaluation of the airway patency & Lumen diameter $\geq 50 \%$ \\
\hline Saturation & $\begin{array}{l}\text { Oxygen saturation (Nonin Medical model } 7500 \text {, } \\
\text { Nonin, Plymouth, Minnesota) }\end{array}$ & $\mathrm{S}_{\mathrm{pO}_{2}}>95 \%$ \\
\hline Level of consciousness & $\begin{array}{l}\text { Level of consciousness assessed by GCS rated by } \\
\text { a senior neurologist }\end{array}$ & $\mathrm{GCS} \geq 8$ \\
\hline
\end{tabular}

\section{Experimental Decannulation Protocol}

This protocol was designed following the guidelines described by Santus et al, ${ }^{6}$ who suggested the evaluation of the following major quantitative parameters: voluntary cough (maximum expiratory pressure; cough peak flow $>160 \mathrm{~L} / \mathrm{min}$ ) and tube capping for $\geq 24 \mathrm{~h}$ ). Moreover, another 8 minor semi-quantitative criteria were considered in the following domains: level of consciousness (drowsy/alert); secretions (thick vs thin); swallowing (impaired vs normal); $\mathrm{CO}_{2}$ levels $\left(\mathrm{P}_{\mathrm{aCO}_{2}}<60 \mathrm{~mm} \mathrm{Hg}\right.$ ); airway patency (tracheal stenosis $<50 \%$ seen by bronchoscopy); age $<70 \mathrm{y}$; indication for tracheostomy; and comorbidities (present vs none). Some of these criteria are based on subjective assessment, depending upon the expertise of the healthcare staff, which is a limitation in the decannulation process. For this reason, we implemented a new protocol including only quantitative parameters to achieve objective evaluation.

This protocol was completed by a senior speech and language therapist, a senior respiratory therapist, and a senior otorhinolaryngologist before the decannulation protocol. It was hypothesized that decannulated subjects were positive for the following selected parameters (Table 1):

1. Presence and efficacy of voluntary cough were assessed based on a study by Bach et al, ${ }^{13}$ in which subjects were evaluated with a spirometer (Pony FX Cosmed, Rome, Italy). Subjects had a negative score when cough peak flow $<160 \mathrm{~L} / \mathrm{min}$ or they could not cough voluntarily due to cognitive deficits (eg, buccofacial apraxia, lack of responsiveness).
2. The cough reflex test consisted of the inhalation of a nebulized tussigenic agent $(0.1 \mathrm{~mol} / \mathrm{L}$ of citric acid $)$, using an ultrasonic nebulizer (MO-03, Norditalia Elettromedicali, San Martino della Battaglia), with a particle size of $3 \mu \mathrm{m}$ and a fixed output rate of $0.9 \mathrm{~mL} / \mathrm{min}$. The inhalation administration was presented via a mouthpiece mask and had a 15-s delivery period, alternated with a 60 -s rest period. Each administration was repeated 3 times. The cough response was considered positive when at least 2 coughs were elicited. ${ }^{14,15}$

3. Tracheostomy tube capping for at least $72 \mathrm{~h}$. This consisted of the ability to keep the tracheostomy stoma closed and breathe though the mouth. This has been described by Stelfox et $\mathrm{al}^{12}$ as a fundamental determinant for tracheostomy decannulation.

4. Swallowing instrumental assessment was carried out through fibro-endoscopic evaluation with liquid and food administrations, reported as the accepted standard for swallowing. ${ }^{16,17}$ Severity was assessed using the penetration aspiration scale (scores 1-8). ${ }^{18}$ A score of 1 indicates absence of dysphagia, scores between 2 and 5 indicate swallowing difficulties and penetration events, and scores $>5$ show aspiration events.

5. The blue dye test consisted of 4 drops of $1 \%$ Evans blue dye on the back of the subject's tongue. The trachea was suctioned at set intervals every $4 \mathrm{~h}$ for $12 \mathrm{~h}$ to verify the presence blue-tinged tracheal secretions, suggesting aspiration. ${ }^{19,20}$

6. The number of tracheal suctions was registered in a table at the subject's bedside. Nurses were instructed to record the numbers of suctions. A negative score was considered as 2 or more suctions every $8 \mathrm{~h}^{21}$ 
7. Instrumental evaluation of patency of the airways was evaluated by endoscopy, considering tracheal stenosis as lumen diameter $<50 \% .4,5,10$

8. $\mathrm{S}_{\mathrm{pO}_{2}}>95 \%$ in ambient air. $6,12,22$

9. Level of consciousness was measured with the Glasgow coma scale $21,23,24$ (GCS, score 3 = totally unresponsive, severe deficit; score $15=$ best responsive, no deficit), rated by a senior neurologist. The level of consciousness was considered insufficient when the GCS was consistently less than 8 (comatose subject).

\section{Follow-Up Decannulation Assessment}

Follow-up decannulation assessment, considered as the reference standard, was carried out $48 \mathrm{~h}$ after tube removal for decannulated subjects. Weaning failure was defined as the failure to pass a spontaneous-breathing trial or the need for recannulation within $48 \mathrm{~h}$ after tube removal.

\section{Data Analysis}

Demographic data were summarized using percentages and means with standard deviations. In the analyses we examined the parameters separately, and then in 3 clusters. Two of the clusters were created to combine the clinical tests considered specific for airway patency (ie, tube capping, instrumental assessment of airways patency) and dysphagia (ie, blue dye test, swallowing instrumental assessment), respectively, and the third was the combination of all 4 parameters (ie, clinical cluster for decannulation). Differences in quantitative variables between subject groups were assessed with Student $t$ test or Mann-Whitney test, according to the Shapiro-Wilk test of normality. Subsequently, sensitivity, specificity, negative predictive value, positive predictive value, positive likehood ratio, negative likehood ratio were calculated to determine the performance of each parameter. For categorical data, the association between 2 variables was tested using the Fisher exact test. A penalized logistic regression analysis, based on Firth's bias reduction method, was used to determine which clusters were associated with successful decannulation at follow-up. Finally, a receiver operating characteristic curve analysis was performed to assess the discrimination ability of the logistic regression. Statistical significance was set at $P<.05$. Data were analyzed using the statistical software R.

\section{Results}

Seventy-four participants (mean age, $51.5 \pm 16.8$ y; 32 females) were recruited; 57 (77\%) of them were decannulated over the 48 -h time period (mean age $54.6 \pm 15.4 \mathrm{y}$ ) and $17(22.9 \%$ ) (mean age $58.8 \pm 14.6$ y) were not. Three (5\%) of the 57 decannulated subjects failed the follow-up

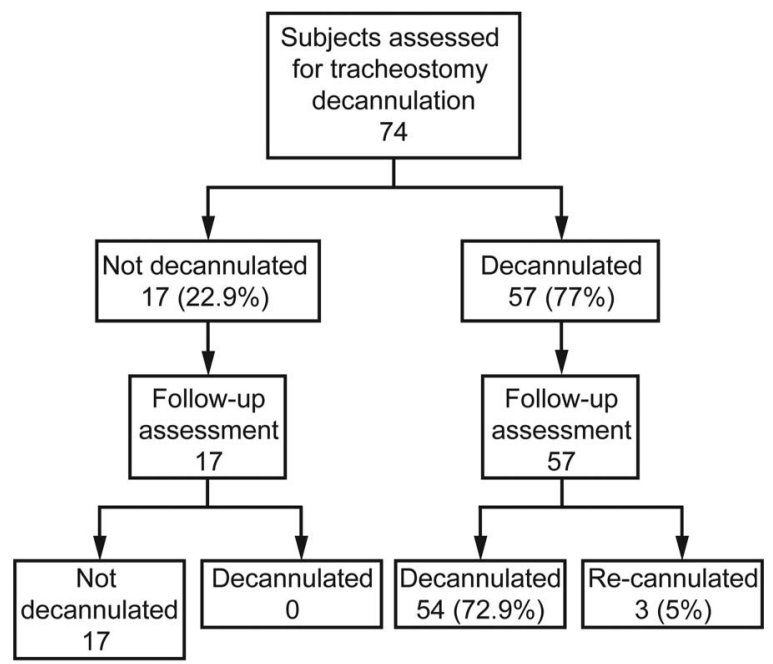

Fig. 1. Flow chart.

assessment after $48 \mathrm{~h}$ and underwent emergency recannulation due to lack of airway patency; 2 subjects presented with tracheomalacia, while 1 subject had bilateral paralysis of the vocal folds (Fig. 1). All other parameters assessed by the protocols were normal, indicating a possible successful decannulation.

Age was not significantly different between decannulated and non-decannulated subject groups $(t=-0.65$, $P=.51)$. Time from cannula placement to assessment was significantly different between decannulated (mean, $66.1 \pm 42.6 \mathrm{~d}$ ) and non-decannulated (mean, $82.5 \pm 97.3 \mathrm{~d}$ ) subjects $(P<.001)$.

Fisher exact test showed that all of the parameters were strongly associated with decannulated and non-decannulated subjects groups (Table 2). Sensitivity and specificity were estimated for all the parameters (Table 2). Parameters showing the best values of sensitivity and specificity were tracheostomy tube capping, swallowing instrumental assessment using penetration aspiration scale scores $\leq 5$, number of tracheal suctions, and the blue dye test. Airway patency, cough reflex test, $\mathrm{S}_{\mathrm{pO}_{2}}$, and GCS $\geq 8$ showed high specificity but low sensitivity. On the contrary, voluntary cough and swallowing instrumental assessment using a penetration aspiration scale score $=1$ showed high sensitivity but low specificity.

In addition, with the aim to improve the ability to detect better tracheal patency, we combined positivity of airway patency and tube-capping tests in a tracheal patency cluster, which resulted in a better sensitivity $(94.1 \%)$ and specificity $(94.7 \%)$ than each test separately. Thus, we considered this as a new reference standard for tracheal patency. We also created a dysphagia cluster including blue dye test and swallowing instrumental assessment penetration aspiration scale scores $\leq 5$ to verify whether dysphagic subjects (without secretion aspiration) were also candidates 


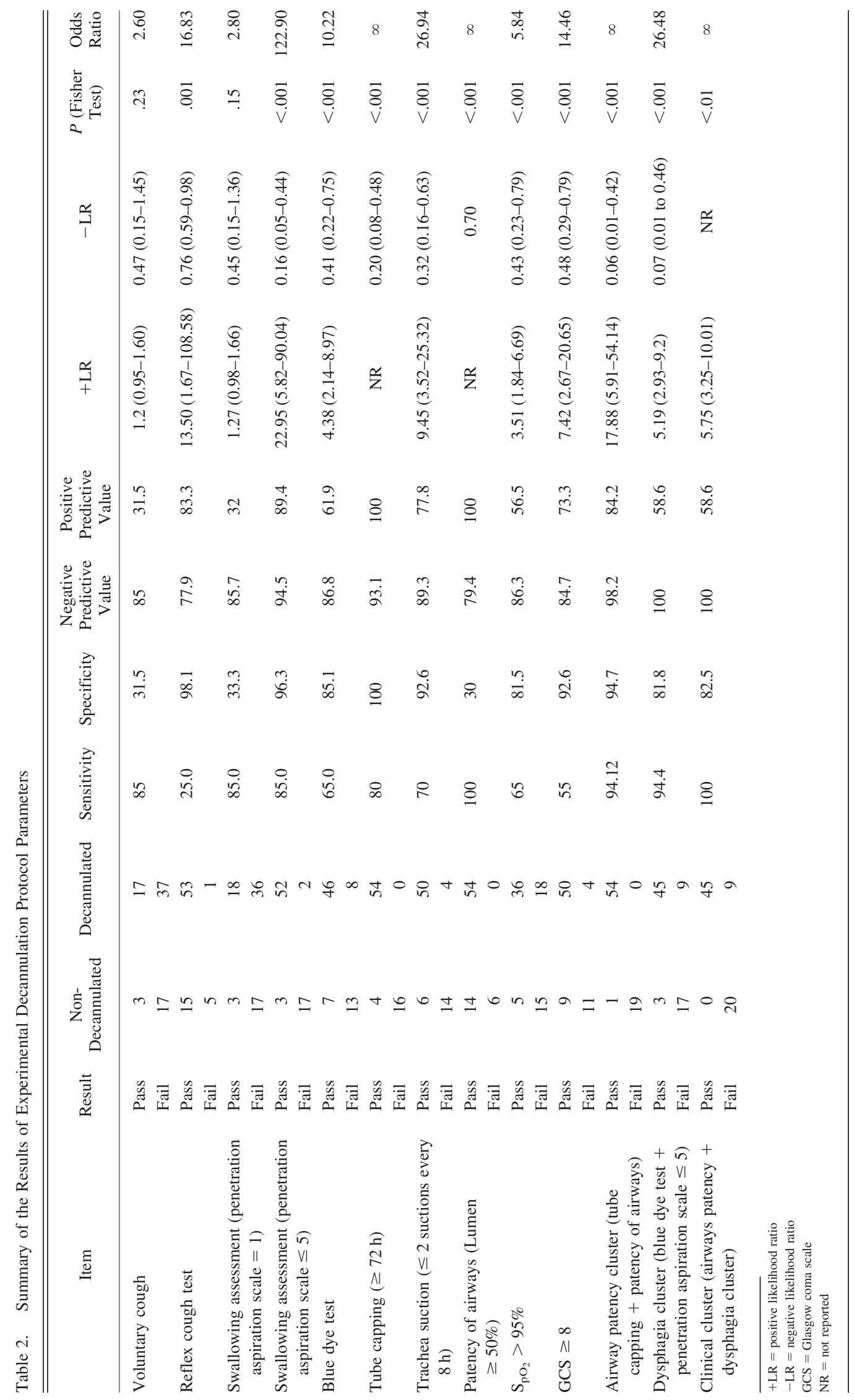


Table 3. Summary of the Penalized Logistic Regression Analyses Using Cluster as Covariates

\begin{tabular}{lccc}
\hline \hline \multicolumn{1}{c}{ Covariate } & $\begin{array}{c}\text { Log(Odds } \\
\text { Ratio }) \pm \mathrm{SD}\end{array}$ & $\begin{array}{c}\text { Correct } \\
\text { Classification } \\
\pm \mathrm{SD}(\%)\end{array}$ & $\begin{array}{c}\text { Area Under the } \\
\text { Curve } \pm \mathrm{SD}\end{array}$ \\
\hline Clinical cluster & $5.28 \pm 1.4$ & 0.88 & 0.92 \\
Dysphagia cluster & $3.17 \pm 0.69$ & 0.84 & 0.84 \\
Patency cluster & $7.26 \pm 1.69$ & 0.98 & 0.98 \\
\hline
\end{tabular}

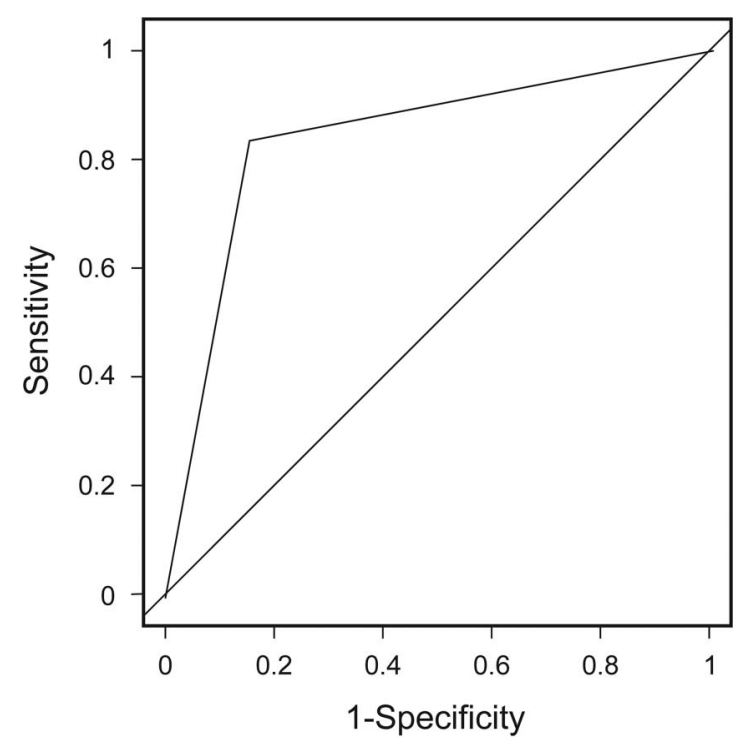

Fig. 2. Receiver operating characteristic curve of the dysphagia cluster, showing an area under the curve of 0.84 .

for successful decannulation. The dysphagia cluster had high sensitivity (94.4\%) and specificity (81.8). Thereafter, the positivity to our new tracheal patency cluster was combined with the dysphagia cluster in a decannulation cluster, which showed a sensitivity of $100 \%$ and specificity of $82.5 \%$.

Penalized logistic regression analyses were fitted using cluster as covariates. ${ }^{26,27}$ The results are given in Table 3 . Receiver operating characteristic curves and area under the curve were computed for each model (Fig. 2, 3, 4). The best model has patency cluster as a covariate, which also has the higher percentage of correct classification (0.98) (Table 3).

\section{Discussion}

We investigated the most significant and objective parameters for tube removal in postacute ABI subjects. From our findings, 4 parameters showed adequate sensitivity and specificity to be clustered as a unique diagnostic test for tracheostomy tube weaning among postacute ABI subjects.

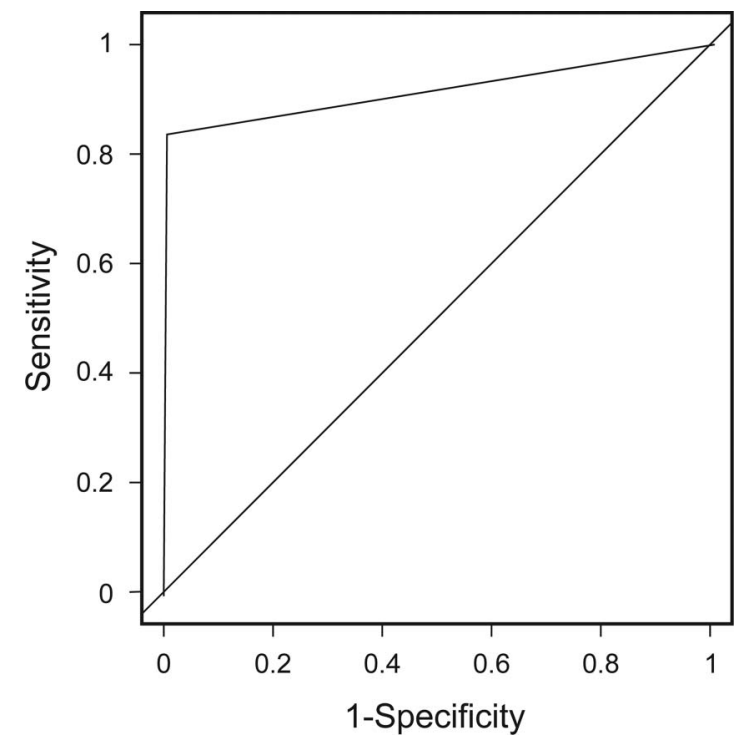

Fig. 3. Receiver operating characteristic curve of the clinical cluster, showing an area under the curve of 0.92 .

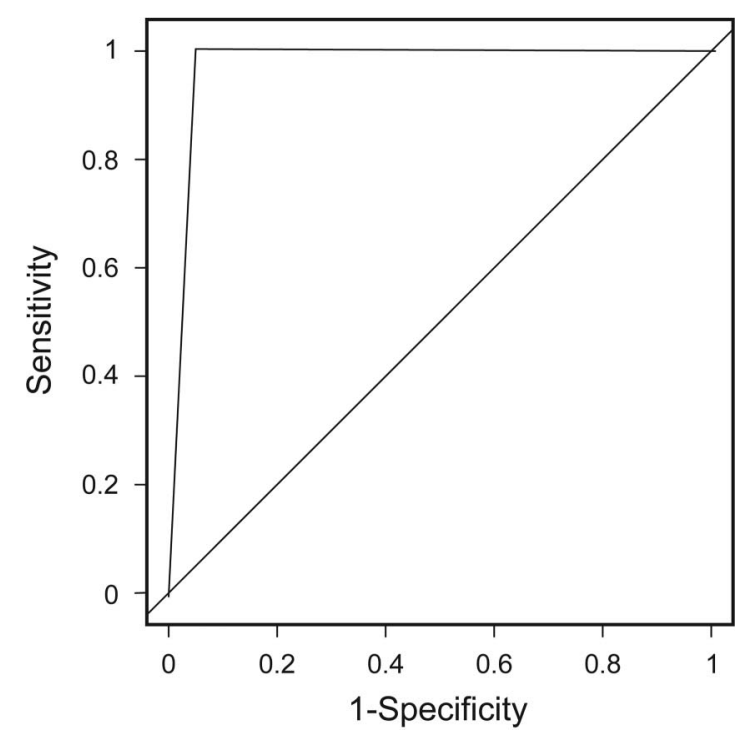

Fig. 4. Receiver operating characteristic curve of the patency cluster, showing an area under the curve of 0.98 .

In the literature, most of the decannulation protocols are designed for acute tracheostomized subjects, who differ significantly from those who are postacute. ${ }^{6}$ Our postacute subjects were more severe, not only because they failed the first decannulation trial, but also because having a tracheostomy tube for a long period of time negatively affects oropharyngeal-laryngeal structures, altering swallowing dynamics and airways structures. ${ }^{4,7}$

A systematic review of tracheostomy decannulation proposed a protocol of 2 quantitative and 8 semi-quantitative criteria for safe decannulation mainly for acute subjects. ${ }^{6}$ Nevertheless, the majority of these selected criteria con- 
sisted of subjective assessments, requiring clinical expertise. Conversely, we included in our study a detailed quantitative evaluation for each parameter. We found that the most sensitive and specific criteria for decannulation were tracheostomy tube capping, instrumental evaluation of patency of the airways, blue dye test, and swallowing instrumental assessment (penetration aspiration scale score $\leq 5$ ).

The tracheostomy tube-capping trial consisted of the ability to breathe through the mouth with the tracheostomy cannula closed with a cap for $72 \mathrm{~h}$. In a study by Santus et al, ${ }^{6}$ the ability to tolerate tracheostomy tube capping and cough effectiveness were the most frequent criteria used by clinicians to predict successful decannulation. Our study confirmed this high sensitivity and specificity, but at follow-up assessment 3 participants were false negative. Specifically, these subjects passed the capping trial test with no saturation reductions, but when the tube was removed they underwent emergency tracheostomy tube replacement because of respiratory failure. Two of these subjects presented with tracheomalacia, and one had bilateral vocal fold paresis. It seems that the tracheostomy tube was keeping the airway open. In the literature, few studies confirmed that a partial upper airway occlusion could be asymptomatic when subjects breathe at tidal volume as occurred in our study. In particular, Law et $\mathrm{al}^{7}$ observed airway lesions in $67 \%$ of subjects with long-term tracheostomy tubes, characterized by tracheal granuloma $(60 \%)$ and tracheomalacia (29\%). Less frequently observed lesions were tracheostenosis (14\%) and vocal cord and laryngeal dysfunction (8\%). We inferred that the tube-capping test does not seem to be a sufficient criterion for airway patency assessment in long-term tracheostomy patients who often present with episodes of obstructive airway lesions. Therefore, in this population, the tube capping should always be executed in combination with an endoscopic evaluation of airway patency. The instrumental assessment of airway patency allows not only the verification of an open tracheal lumen, but also the presence of edema, granuloma, and tropism of trachea tissue, which could occlude the airways. ${ }^{4,7}$ For this reason, we created a clinical cluster including the tube capping and endoscopic evaluation of airway patency. This criterion reached higher specificity and sensitivity, confirming the importance of both tests for the assessment of airway patency in a decannulation protocol.

In our study, another fundamental criterion was the fibro-endoscopic swallowing assessment, which allowed the detection of the presence or absence of dysphagia ${ }^{18}$ and the severity of swallowing disorders, verifying the presence of food or secretions in airways (penetration aspiration scale score $=1$ indicates absence of dysphagia; penetration aspiration scale score $=2-5$ indicates penetrations; penetration aspiration scale scores $=5-8$ indicates aspiration). In the literature, many studies reported only the presence or absence of dysphagia assessed by a subjective clinical evaluation, ${ }^{6}$ depending upon clinician experience, without any information on inhalations. Interestingly, we found that the presence of mild dysphagia characterized by pharyngeal residues or penetration (penetration aspiration scale scores 2-5) was not a negative predictor of decannulation. Indeed, tubes were removed from subjects with mild dysphagia without aspiration who had good management of saliva (penetration aspiration scale score $\leq 5$ ). This result contrasts with a previous study ${ }^{6,11}$ that evaluated generic dysphagia symptoms and revealed the importance of penetration/aspiration examinations during tracheostomy tube removal. Clinically, the presence of inhalation is often assessed by the blue dye test, ${ }^{19}$ which is well known for its high specificity and sensitivity. Our results confirmed that the presence of blue-dyed secretions in the inner cannula is an indicator of aspiration of secretions into the airways and is a negative predictor of decannulation success. However, the blue dye test does not give information on the presence and localization of pharyngeal residues or the potential causes of inhalations. We combined fibro-endoscopic evaluation with the blue dye test in a dysphagia cluster, which showed higher specificity and sensitivity. For this reason, we recommend combining these in neurologic postacute patients in order to have complete information on swallowing function.

On the basis of these results for both airway and swallowing assessments, we created a clinical prediction rule to identify the best combination parameters for decannulation. We included the cluster for airway patency and the dysphagia cluster. The combination of these parameters had high sensitivity and specificity, demonstrating the importance of airway patency and secretion management evaluation. This does not imply that other tests are not important; however, the tube-capping trial, tracheal lumen $>50 \%$ evaluated by endoscopy, fibro-endoscopic swallowing assessment (penetration aspiration scale score $\leq 5$ ), and blue dye test are likely to be essential evaluations for tube removal in severe neurological patients.

There is broad consensus that voluntary cough efficiency $^{13}$ is one of the most predictive criteria for decannulation. In contrast, our results show that the assessment of voluntary cough was not sufficient; the sensitivity was high but the specificity was too low. This result depends on the difficulties that many neurological patients with severe cognitive deficits have in performing voluntary cough. ${ }^{24}$ Subjects with severe ABI often could not answer simple commands (eg, "try to cough") not only because of cognitive impairment but also due to buccofacial apraxia that compromised the execution of voluntary cough. ${ }^{15}$ The voluntary cough assessment, which requires cortical control and volitional drive, is different from the cough reflex, which is triggered by laryngeal tussigenic receptors. ${ }^{15}$ For this reason, we tested the reflex cough 
using a nebulized tussigenic, which does not require any voluntary interventions by patients. The cough reflex test was administered to all subjects and showed high specificity for tracheostomy tube removal. Hence, the reflex cough test seems to be more appropriate for this population, providing information on involuntary mechanisms of airway protection from inhaled secretions, although it is not a sufficient criterion for decannulation.

Another criterion for assessing secretion management is to count the number of tracheal suctions. ${ }^{21}$ In our study, this criterion was sensitive and specific. It is, however, too subjective because it is dependent on the healthcare professionals and caregivers who are responsible for the patient. In addition, there are now methods of mechanical and manual cough assist that are designed to remove secretions, so it seems that computing the number of tracheal-suctions is not an essential evaluation for decannulation decisions.

We also analyzed the level of consciousness and oxygen saturation criteria as suggested by Santus et al. ${ }^{6}$ They defined the level of consciousness as alert versus drowsy but aroused status. Nevertheless, we decided to fix alert status at GCS $\geq 8$, corresponding to a non-comatose status. ${ }^{23}$ In our study, 7 (9.4\%) subjects with GCS $<8$ were successfully decannulated because they swallowed saliva efficiently and were fed only via percutaneous endoscopic gastrostomy, although they were in a semi-comatose status $(\mathrm{GCS}=5)$. In contrast with Santus et al, ${ }^{6}$ we inferred that the level of consciousness was not fundamental for decannulation in $\mathrm{ABI}$ patients who were fed by percutaneous endoscopic gastrostomy and had good management of secretions.

Likewise, oxygen saturation was not a predictable parameter for decannulation. Most decannulated subjects had $\mathrm{S}_{\mathrm{pO}_{2}}>95 \%$, and $11 \%$ had low saturation peak events $<90 \%$, but they were successfully decannulated because the saturation was stabilized by the use of noninvasive ventilation or oxygen therapy. 25

The major limitation of the our study was the significant difference in the time from tracheostomy tube placement to assessment among decannulated and non-decannulated subjects. We are aware that non-decannulated subjects had the tube for a longer period than decannulated subjects, which could have impacted the weaning process negatively. However, the positive decannulation group had the tracheostomy tube for longer periods (mean $66.1 \pm 42.6 \mathrm{~d}$ ) than has been reported in literature, so these data increase knowledge on decannulation and the period of time of tracheostomy tubes stay in place.

\section{Conclusions}

These results suggest that the clinical prediction rule for decannulation in ABI patients is the combination of the following assessments: (1) tracheostomy tube capping, (2) endoscopic assessment of patency of airways, (3) swallowing instrumental assessment using penetration aspiration scale scores $\leq 5$, and (4) blue dye test. Nevertheless, further evaluations such as voluntary and reflex cough and level of consciousness yield important information on patients, although they are not essential parameters for decannulation in persons with ABI. Decannulation as quickly and safely as possible should be the primary goal of the multidisciplinary team ${ }^{28}$ in neurologic rehabilitation to increase recovery and the independence.

\section{ACKNOWLEDGMENT}

We would like to thank Dr Elisabetta Gasparoli, Associazione Riabilitatori dell'Insufficienza Respiratoria, for her help in conceiving the study and giving suggestions, as well as the Respiratory Physiotherapists and Speech and Language Therapists, and the team at the Rehabilitation Unit (Fondazione Ospedale di Neuroriabilitazione, Istituto di Ricovero e Cura a Carattere Scientifico, San Camillo).

\section{REFERENCES}

1. Durbin CG. Indications for and timing of tracheostomy. Respir Care 2005;50(4):483-487.

2. Durbin CG Jr. Tracheostomy: why, when, and how? Respir Care 2010;55(8):1056-1068.

3. Christopher KL. Tracheostomy decannulation. Respir Care 2005; 50(4):538-541.

4. Chadda K, Louis B, Benaissa L. Physiological effects of decannulation in tracheostomized subjects. Intensive Care Med 2002;28(12): 1761-1767.

5. O'Connor HH, White AC. Tracheostomy decannulation. Respir Care 2010;55(8):1076-1081.

6. Santus P, Gramegna A, Radovanovic D, Raccanelli R, Valenti V, Rabbiosi D, et al. A systematic review on tracheostomy decannulation: a proposal of a quantitative semiquantitative clinical score. BMC Pulmonary Medicine 2014;14(1):201-209.

7. Law JH, Barnhart K, Rowlett W, de la Rocha O, Lowenberg S. Increased frequency of obstructive airway abnormalities with longterm tracheostomy. Chest 1993;104(1):136-8.

8. Engels PT, Bagshaw SM, Meier M, Brindley PG. Tracheostomy: from insertion to decannulation. Can J Surg 2009;52(5):427-433.

9. Clini E, Vitacca M, Bianchi L, Porta R, Ambrosino N. Long-term tracheostomy in severe COPD subjects weaned from mechanical ventilation. Respir Care 1999;44(4):415-420.

10. Ceriana P, Carlucci A, Navalesi P, Rampulla C, Delmastro M, Piaggi G, De Mattia E, Nava S. Weaning from tracheotomy in long-term mechanically ventilated subjects: feasibility of a decisional flowchart and clinical outcome. Intensive Care Med 2003;29(5):845-848.

11. Garuti G, Reverberi C, Briganti A, Massobrio M, Lombardi F, Lusuardi M. Swallowing disorders in tracheostomised subjects: a multidisciplinary/multiprofessional approach in decannulation protocols. Multidiscip Respir Med. 2014;9(1):36-46.

12. Stelfox HT, Crimi C, Berra L, Noto A, Schmidt U, Bigatello LM, Hess D. Determinants of tracheostomy decannulation: an international survey. Critical Care 2008;12(1):R26.

13. Bach JR, Saporito LR. Indications and criteria for decannulation and transition from invasive to noninvasive long-term ventilatory support. Respir Care 1994;39(5):515-528.

14. Battel I, Ceolin A, Koch I, Ventura L, Tonin P, Palmer K, Meneghello F, Comparison of the cough reflex test and water swallow- 


\section{Clinical Criteria for Tracheostomy Decannulation}

ing test in healthy participants and neurological subjects. B-ENT 2016;4(12):285-289.

15. Morice AH, Fontana GA, Belvisi MG, Birring SS, Chung KF, Dicpinigaitis PV, et al. ERS guidelines on the assessment of cough. Eur Respir J 2007;29(6):1256-76.

16. Butler SG, Markley L, Sanders B, Stuart A. Reliability of the penetration aspiration scale with flexible endoscopic evaluation of swallowing. Ann Otol Rhinol Laryngol 2015;124(6):480-3.

17. Cameron JL, Reynolds J, Zuidema GD. Aspiration in subjects with tracheotomies. Surg Gynecol Obstet 1973;136(1):68-70.

18. Rosenbek JC, Robbins JA, Roecker EB, Coyle JL, Wood JL. A penetration-aspiration scale. Dysphagia 1996;11(2):93-8.

19. O'Neil-Pirozzi TM, Lisiecki DJ, Jack Momose K, Connors JJ, Milliner MP. Simultaneous modified barium swallow and blue dye tests: A determination of the accuracy of blue dye test aspiration findings. Dysphagia 2003;18(1):32-38.

20. Béchet S, Hill F, Gilheaney Ó, Walshe M. Diagnostic accuracy of the modified Evan's blue dye test in detecting aspiration in subjects with tracheostomy: a systematic review of the evidence. Dysphagia 2016;31(6):721-729.

21. Martinez GH, Fernandez R, Casado MS, Cuena R, Lopez-Reina P, Zamora S, Luzon E. Tracheostomy tube in place at intensive care unit discharge is associated with increased ward mortality. Respir Care 2009;54(12):1644-1652.

22. Marchese S, Corrado A, Scala R, Corrao S, Ambrosino N. Tracheostomy in patients with long-term mechanical ventilation: a survey. Respir Med 2010;104(5):749-753.

23. Teasdale G, Jennett B, Murray L, Murray G. Glasgow coma scale: to sum or not sum. The Lancet 1983;2(8351):678-678.

24. Zanata Ide L, Santos RS, Hirata GC. Tracheal decannulation protocol in subjects affected by traumatic brain injury. Int Arch Otorhinolaryngol 2014;18(2):108-114.

25. Bach JR, Saporito LR, Shah HR, Sinquee D. Decannulation of subjects with severe respiratory muscle insufficiency: efficacy of mechanical insufflation-exsufflation. J Rehabil Med 2014;46(10):1037-41.

26. Heinze G, Schemper M. A solution to the problem of separation in logistic regression. Stat Med 2002;21(6):2409-2419.

27. Heinze G. A comparative investigation of methods for logistic regression with separated or nearly separated data. Stat Med 2006; 25(24):4216-4226.

28. Frank U, Mäder M, Sticher H. Dysphagic subjects with tracheotomies: a multidisciplinary approach to treatment and decannulation management. Dysphagia 2007;22(1):20-9.

This article is approved for Continuing Respiratory Care Education credit. For information and to obtain your CRCE

(free to AARC members) visit

www.rcjournal.com 